\title{
Neuropsychological outcome in cases with acute disseminated encephalomyelitis
}

\author{
Seda Kanmaz ${ }^{1 \oplus}$, Sezen Köse ${ }^{2 \oplus}$, Cenk Eraslan ${ }^{3 \oplus}$, Erdem Şimşek ${ }^{1 \oplus,}$ \\ Hepsen Mine Serin ${ }^{1 \oplus}$, Sanem Yılmaz ${ }^{1 \oplus}$, Gül $\operatorname{Aktan}^{1 \oplus}$, Hasan Tekgül $^{1 \oplus}$, \\ Hatice Serpil Erermiş ${ }^{2 \oplus}$, Sarenur Gökben ${ }^{1 \oplus}$ \\ ${ }^{1}$ Division of Child Neurology, Department of Pediatrics, ${ }^{2}$ Child and Adolescent Psychiatry, ${ }^{3}$ Neuroradiology, Ege University Faculty of \\ Medicine, İzmir, Turkey.
}

\begin{abstract}
Background and objectives. Acute disseminated encephalomyelitis (ADEM) is an immune-mediated, inflammatory and demyelinating disorder of the central nervous system. There have been a few studies in recent years on the fact that these cases have neurocognitive impairment. The purpose of this study is to evaluate the neurocognitive outcome and quality of life in cases with ADEM.

Methods. Eleven cases who were on follow-up between 2008 and 2017 were included in the study, systemic, neurological and psychiatric examinations were done. All magnetic resonance images were re-evaluated. The neuropsychiatric evaluation was performed by clinical examination and psychometric scales; (1) The Pediatric Quality of Life Inventory 4.0, (2) Child Behavior Checklist, (3) Children's Depression Inventory, (4) The Wechsler Intelligence Scale for Children-Revised and (5) Continuous Performance Test. The cases in our study underwent neuropsychiatric evaluation 3-42 months after the diagnosis of ADEM had been established.
\end{abstract}

Results. Nine cases $(81.8 \%)$ fully recovered without neurologic deficit. One case $(9.1 \%)$ had a psychiatric disorder. During follow-up, cognitive and psychiatric problems were encountered in half of the cases (54.5\%). Most of the cases with basal ganglia involvement $(80 \%)$ displayed attention deficit and cognitive problems.

Conclusion. In particular, cases with basal ganglia involvement should be followed carefully in terms of attention and cognitive problems.

Key words: ADEM, neuroimaging, neuropsychiatric disorders.

Acute disseminated encephalomyelitis (ADEM) is an immune-mediated, inflammatory and demyelinating disorder of the central nervous system (CNS). It is characterized by multifocal neurological symptoms and encephalopathy and is often triggered by an infection or vaccination. ${ }^{1,2}$ It is more common in males and in children aged 5 to 8 years with an incidence of $0.3-0.6 / 100,000 .^{3}$ The diagnosis of ADEM is based upon clinical and abnormal craniospinal magnetic resonance imaging (MRI) findings.

\footnotetext{
Seda Kanmaz

drsedakanmaz@gmail.com
}

Received 19th October 2019, revised 26th December 2019, accepted 15th January 2020.
Cranial MRI findings are characterized by diffuse, large, poorly marginated lesions in the white matter, best observed in T2-weighted and fluid-attenuated inversion recovery (FLAIR) sequences, but the gray matter can also be involved. ${ }^{4}$ It has been reported that the size or location of the lesions at the beginning are not related with the outcome. ${ }^{5-7}$ Although most cases recover completely, residual neurological problems (motor deficit, dysphasia, reduced visual acuity, ataxia, and epilepsy) can be observed in some cases. ${ }^{5,8}$ In recent years, neuropsychiatric symptoms such as cognitive impairment, behavioral disorders, and attention deficit have been reported in cases diagnosed with ADEM, but there are only a few studies in this subject. ${ }^{9-15}$ The purpose of this study is 
to evaluate the neurocognitive outcome and quality of life in cases with ADEM, also to examine the relationship between the data and the clinical and radiological features.

\section{Material and Methods}

\section{Participants}

Twenty seven cases were diagnosed with ADEM and treated between January 2008 and December 2017 in the Child Neurology Department at Ege University Hospital. Diagnosis of ADEM depending on both clinical (multifocal CNS involvement besides encephalopathy) and radiological findings was established. During follow-up five cases showed new attacks, they were diagnosed with multiple sclerosis or neuromyelitis optica spectrum diseases, depending on clinical, radiological and biochemical findings. All of them were excluded. The cases with the inclusion criteria described below were recruited from 22 cases with ADEM.

The study inclusion criteria were:

1) Cases who were diagnosed with monophasic ADEM.

2) Cases who were aged 6-16 years at the time of study for the availability of normative data for the neuropsychologic tests.

3) Cases who were followed up at least for the first three months after the ADEM diagnosis.

Five cases who were diagnosed with ADEM previously could not be reached since their contact details had changed. Six of 22 cases with ADEM were excluded since they were younger than 6 years or older than 16 years at the time of study. Eleven cases were enrolled in the study.

\section{Procedures}

The cases were contacted by telephone and were invited to our clinic for neurocognitive and psychiatric evaluation. The case's complaints were inquired by the pediatric neurologist, systemic and neurological examinations were performed. Gender, age at the time of diagnosis, presenting symptoms, duration of treatment and follow-up duration were recorded from the case files. Craniospinal MRI scans performed at beginning of the illness were available for all the cases. Follow-up of neuroimages were obtained between $3^{\text {rd }}$ month- $2^{\text {nd }}$ years. First and last follow-up MRI scans of the cases were reevaluated by the neuroradiologist. Radiological assessment was recorded according to the criteria described below: ${ }^{16}$

1) Areas of involvement: white matter, gray matter, deep gray matter (basal ganglia, thalami), brain stem, spinal cord

2) Enhancement administration of gadolinium

3) The presence of mass effect

Follow-up MRI findings were described as complete resolution (disappearance of all demyelinating lesions) and partial resolution (decrease in size or signal intensity of demyelinating lesions).

\section{Cognitive and Neuropsychological Assessment}

Schedule for Affective Disorders and Schizophrenia for School-Aged Children, Present and Lifetime Version (K-SADS-PL) is a semi-structured interview used to determine psychopathology in children and adolescents according to DSM-IV-TR criteria. ${ }^{17}$ The Turkish adaptation study was conducted by Gökler et al. ${ }^{18}$ The clinician-rated the score according to the interview conducted with the parent and the child and finally achieved summary ratings using the best clinical judgment.

\section{Wechsler Intelligence Scale for Children-} Revised (WISC-R) is a standardized intelligence test developed by Wechsler (1974) for children which includes two parts; verbal and performance subtests. The total intelligence quotient (IQ) score is calculated by summing verbal and performance scores. The Turkish standardization was conducted by Savaşır and 
Şahin (1995) for children between 6-16 years of age. ${ }^{19}$ Total IQ scores less than 70 was accepted as intellectual disability. Total IQ scores ranged between 70-79 and 80-89 were accepted as borderline intellectual functioning and below average intelligence, respectively. Wechsler (1974) stated that difference between verbal and performance IQ scores of at least 15 points is required to necessitate deeper investigation. ${ }^{20}$

Children's Depression Inventory (CDI) was developed by Kovacs (1985), and it is a 27item self-report questionnaire used to evaluate depressive symptoms of children aged 6-17 years old. The cut-off point for depression is 19; scores higher than 14 can be used for evaluating the children who may be at risk for developing depression. Turkish validation was conducted by Öy for children older than 7 years. ${ }^{21}$

Child Behavior Checklist (CBCL) is a parentrated scale developed to assess competence and problem behaviors in children by Achenbach and Edelbrock. ${ }^{22}$ The Turkish version of the CBCL contains 20 competence items and 118 problem items for children aged between 4 and 18 years. The competence scales of the CBCL are Activity, Social, and School. From these three scales, a Total Competence score is obtained. The problems scored with the checklist are Aggressive Behavior, Anxious/Depressed, Attention Problems, Delinquent Behavior, Social Problems, Somatic Complaints, Thought Problems, and Withdrawn/Depressed. The 118 problem items describe a wide array of problems that are rated on a 3-point scale. Parents score each item as 0,1 , or 2 (not true, somewhat true, or very true, respectively). Scores of all problem scales constitute the total problem and internalizing and externalizing problem scores. A higher score represents higher severity. Children who score 0-66 are considered to be without behavioral or developmental problems.

The Pediatric Quality of Life Inventory 4.0 (PedsQLTM 4.0) is one of the measures that evaluate health-related quality of life (HRQoL) in 2-18-year-old children and adolescents and was developed by Varni, Seid, and Rode. It is a 23-item questionnaire, and takes approximately 7-10 min to complete. It examines four distinct areas of health-related functioning: physical functioning, emotional functioning, social functioning, and school functioning. The latter three scales are combined to determine a broad psychosocial summary score. The scale is composed of a child self-report and parallel parent proxy report that assess parents' perception of their children's HRQoL for ages $5-7,8-12$, and $13-18$ years. The response scale is a 5-point Likert scale. The items are reverse scored between 0 and 100, where the answer " 0 = never a problem" scores 100 and " $4=$ almost always a problem" scores 0 , so that the higher the total PedsQLTM 4.0 score, the better the HRQoL. ${ }^{23}$ Turkish translation, reliability, and validity studies for 2-18 years of children have been carried out. ${ }^{24,25}$

\section{The Conners Continuous Performance} Test Third Edition ${ }^{\mathrm{TM}}$ (Conners CPT $3^{\mathrm{TM}}$ ) is a computer-based program for measuring attention-related problems in individuals aged eight years and older. By indexing the respondent's performance in areas of inattentiveness, impulsivity, sustained attention, and vigilance, the Conners CPT 3 can aid in the assessment of Attention-Deficit/ Hyperactive Disorder (ADHD) and other neurological conditions related to attention. The Conners CPT 3 provides objective information about an individual's performance in attention tasks. The administration of the test takes 14 minutes. The stimuli consist of letters; nontargets (the letter $X$ ) to targets (all other letters). The children are asked to press the space bar or click the mouse for every letter that appears on the screen, except the letter X. Conners CPT3 reports the results on omission and commission rates, overall hit reaction time (Hit RT), overall hit reaction time standard error (Hit RT SE), variability, detectability $\left(\mathrm{d}^{\prime}\right)$, response style $(\beta)$, perseverations, hit reaction time by block change (Hit RT Block Change), hit reaction time standard error by block change (Hit SE Block Change), hit reaction time by interstimulus interval change (Hit RT ISI Change), and hit 
reaction time standard error by interstimulus interval change (Hit SE ISI Change). Conners CPT3 measures are grouped into indicators of inattentiveness, impulsivity, sustained attention and vigilance. For children under the age of 8 years The Kiddie version of the test was used (The Kiddie CPT 2). In the Kiddie version the targets are pictures of objects that are familiar to preschool children such as house or boat, and the non-target stimuli is a ball. ${ }^{26}$

Informed consent was obtained from the cases and their parents. This study was approved by the local ethics committee (18-10.1/4416.10.2018). Statistical analysis was descriptive.

\section{Results}

\section{Neurological Outcome}

Six cases were male and five were female. The mean age at the time of diagnosis was $7.36 \pm 4$ (1-15) years. Three cases were diagnosed with ADEM before the age of five years and eight cases were diagnosed after the age of five years. All cases were polysymptomatic. At the time of diagnosis, the most common findings were altered state of consciousness $(n=8,72.7 \%)$, headache $(n=7,63.6 \%)$ and ataxia $(n=6,54.5 \%)$, respectively. Lumbar puncture was performed in seven cases; cerebrospinal fluid protein level was elevated in one case and four cases had pleocytosis. Cerebrospinal fluid oligoclonal band and anti-myelin oligodendrocyte glycoprotein antibody were evaluated and found as negative in six and three cases, respectively. The mean duration of follow-up was $25 \pm 22$ (3-42) months. Four cases were followed-up for less than one year, whereas seven were followed-up for more than one year. Nine cases $(81.8 \%)$ fully recovered without neurologic deficit during the follow-up period. One case who had a history of respiratory arrest at the time of initial diagnosis and who was being followed for three months had nasal speech and one case had urinary incontinence. Clinic characteristics of the cases are shown in Table I.

\section{Radiologic outcome}

At the time of initial diagnosis, ten cases had $(90.9 \%)$ white matter, six cases $(54.5 \%)$ had cortical, seven cases (63.6\%) had deep gray matter (5:basal ganglia, 6:thalami) six cases $(54.5 \%)$ had brain stem, one case $(9.1 \%)$ had cerebellar and four cases $(36.4 \%)$ had spinal involvement. Mass effect was not found in any of the cases. Seven cases (63.6\%) displayed complete resolution of demyelinating lesions but four cases $(36.4 \%)$ showed decrease in size of the lesions (Table I-II). Only one of four cases having partial resolution had more than one year of follow-up.

\section{Psychiatric and psychometric outcome}

In the psychiatric evaluation of 11 cases, 9 $(81.8 \%)$ had no psychiatric disease, whereas one case (case no 5, female, 16 years old) had a depressive disorder, generalized anxiety disorder (GAD) and social phobia (SP), and one case (case no 3, male, 7 years old) had an articulation disorder.

The case having depressive disorder, GAD and SP was diagnosed with ADEM at the age of 13 years 8 months. Although she had some psychiatric problems, her last neurologic examination and cranial MRI were normal. She has been followed for 30 months since ADEM. When we evaluated this case she was suffering from depression, GAD and SP since 8 months and was taking antidepressant medications. Although medical treatment led to a significant remission of depressive symptoms, GAD and SP disorders were still ongoing. Her CDI score was 33. Although her psychiatric history revealed that she was socially anxious, avoidant and sensitive to separation during childhood, her clinical functionality was not impaired until the age of 15 years, when her sister got married and moved to another city our case had felt lonely. Our case and her mother described that she was fond of her sister. Although psychosocial stressors might cause depressive disorders, the role of ADEM in psychiatric vulnerability is not clear. 


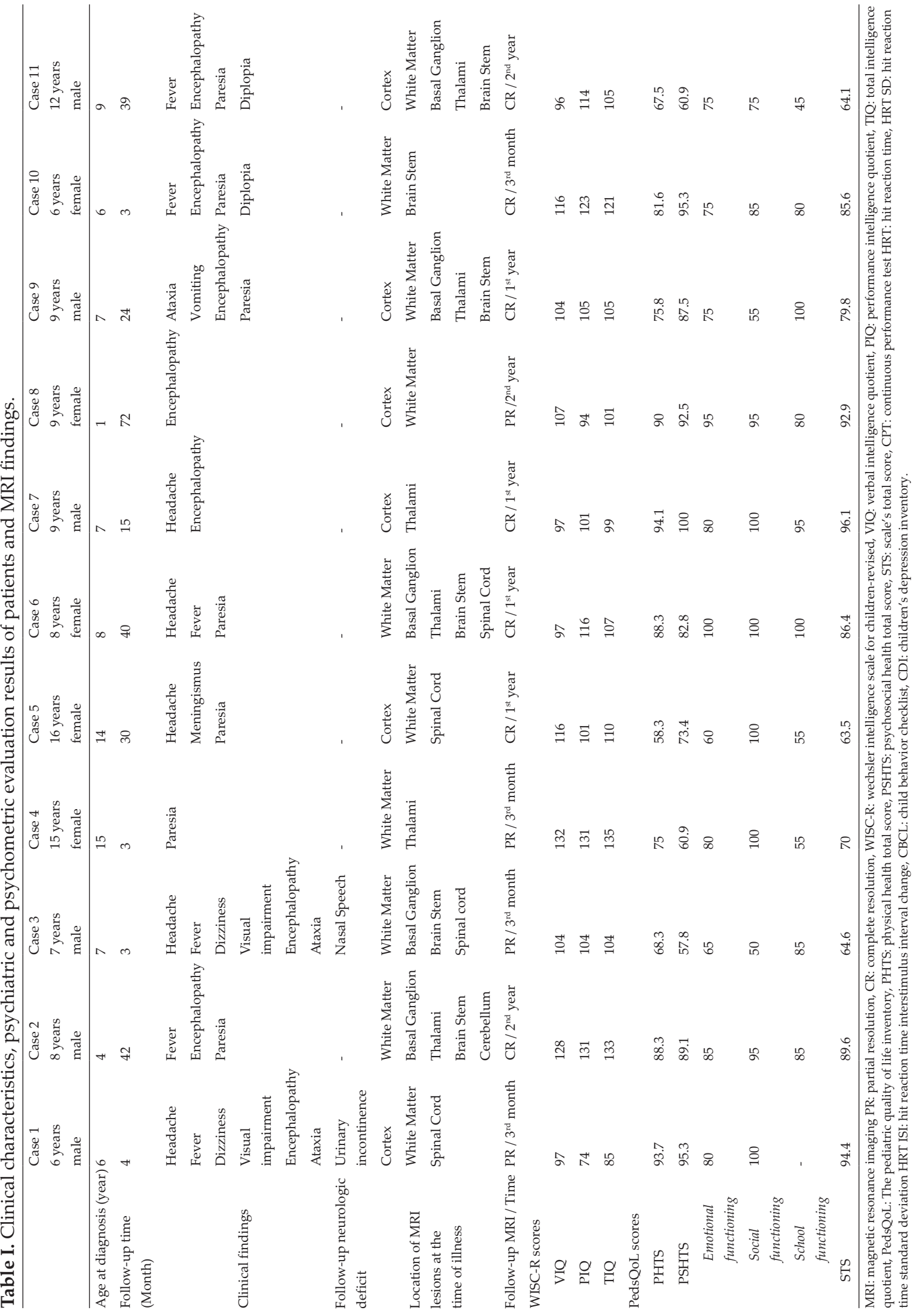











The male case having articulation disorder was diagnosed with ADEM at the age of 7 years and was followed for 3 months. Although articulation disorder was observed during his psychiatric interview, his family reported that it was found before the ADEM attack.

According to the results of the WISC-R intelligence test, only one case (Case1) had borderline intellectual functioning, other cases had average or higher scores (Table I). Two cases (Case 1 and Case 5) demonstrated a lower performance IQ (PIQ) score than verbal IQ (VIQ) score. Case 1 received the lowest score in the coding subtest of PIQ evaluation. Two of the other cases (Case 6 and Case 11) demonstrated lower VIQ score than PIQ score. They received the lowest scores in the information subtest and they also had low scores in coding and block design subtests of PIQ which were determinant of reduced attention and visuospatial/ visuomotor skills. Regarding this result, attention problems were found in 4 of 11 cases $(36.4 \%)$.

The CPT scores of cases are presented in Table I. Seven of eleven cases completed the CPT test. Four cases did not accept to participate in the tests. The T-scores of CPT of those four cases were within the typical range. Two cases (Case1,3) showed only one atypical T-score means and responded more slowly and one case (Case 2) scores showed some atypical $\mathrm{T}$-scores in sustained attention, vigilance and inattentiveness dimensions of attention.

Although CPT results were compatible with attention problems in those cases, their psychiatric evaluations, parent and teacher report forms did not support clinical ADHD diagnosis according to DSM-IV-TR.

Child behavior checklist scores of ten cases were found to be within normal limits, whereas anxiety/depression, social problems, thought problems, attention problems, delinquent behavior, aggressive behavior and internalizing, externalizing and total behavior problem scores were high in one case (Case 1). Although an 
emotional-behavioral problem was defined in the CBCL scale for this case, no active clinical psychopathology was determined on psychiatric examination according to DSMIV-TR. This case was diagnosed with ADEM three months prior and has been within the early follow-up period. For this reason these problems were considered to be associated with the acute course of the disease.

Quality of life scale for children; the mean total scale score, total physical health score, and total psychosocial health score are presented in Table I. Age at time of diagnosis, duration of followup, and initial and control MRI findings of cases were compared with mean PedsQoL scores; however, no statistically significant correlation was found ( $p>0.05)$.

The relationship between first MRI findings and presence of neuropsychological symptoms was also evaluated. The rate of neuropsychological symptoms were the highest in cases that had basal ganglia involvement when compared to cases with cortical, thalamic and brainstem involvement respectively ( $80 \%$ vs $50 \%, 50 \%, 66 \%)$. The rate of partial resolution of lesions in cases with or without neuropsychological symptoms were $33 \%$ and $40 \%$ respectively.

\section{Discussion}

ADEM is an immune-mediated, monophasic demyelinating disorder of the CNS with a good outcome. Less than $10 \%$ of cases may have significant neuropsychiatric morbidity such as motor deficits, focal epilepsy, decreased visual acuity and decline in neurocognitive functions. ${ }^{27}$ In our study, most cases $(81.8 \%$ ) fully recovered without neurological deficit. One case had nasal speech and one case had urinary incontinence. However recent studies have reported that the cases may display permanent sequelae such as behavioral and attention deficit disorders. ${ }^{3,9,15,27}$ These studies showed similar results and are outlined in Table III..$^{10-12,15}$ In our study, psychiatric/neurocognitive impairment was detected in six (54.5\%) cases, two of them also had neurological deficits. One case $(9.1 \%)$ had a psychiatric diagnosis (depressive disorder, generalized anxiety disorder, social phobia), one case $(9.1 \%)$ had behavioral problems, two cases $(18.2 \%)$ had lower PIQ scores than VIQ scores, two cases $(18.2 \%)$ had lower VIQ scores than PIQ scores. Three of seven (42.9\%) cases displayed impaired attention based on CPT.

Attention problems were described in $11-58 \%$ of cases following ADEM in different studies. ${ }^{27-31}$ In our study, two families complained about the lack of attention in their children; but, objective psychiatric examination, family and teacher interviews were not compatible with ADHD. On the other hand, three of 7 (42.9\%) cases had atypical T-scores in sustained attention, vigilance and inattentiveness dimensions of attention in CPT, our results are compatible with literature data. Although three cases did not fulfill the DSM-IV for ADHD, their parents were given information about the results of the $\mathrm{CPT}$ and suggested to follow their children for the symptoms of attention deficits.

The frequency of neurocognitive disorders including borderline IQ, attention deficit, decreased executive functions and psychiatric symptoms following ADEM were also reported between $4-66 \%{ }^{10,12,27-33}$ The results of studies about the relationship between neurocognitive outcomes and the duration of follow-up are conflicting. Some reports point out that longer duration of follow-up is associated with better outcomes and resolving of abnormal neurocognitive findings..$^{27,32}$ Our cases underwent neuropsychiatric evaluation 3-42 months after the diagnosis of ADEM. In six cases neuropsychiatric symptoms were detected, four of them were followed up longer than one year, besides two of them were followed less than one year.

We detected reduced attention and visuospatial/ visuomotor skills in 4 of 11 cases. Two of the them (Cases 1 and 5) demonstrated lower PIQ score than VIQ score, similar to Hahn et al.s'10 study, indicating a cognitive profile of reduced 


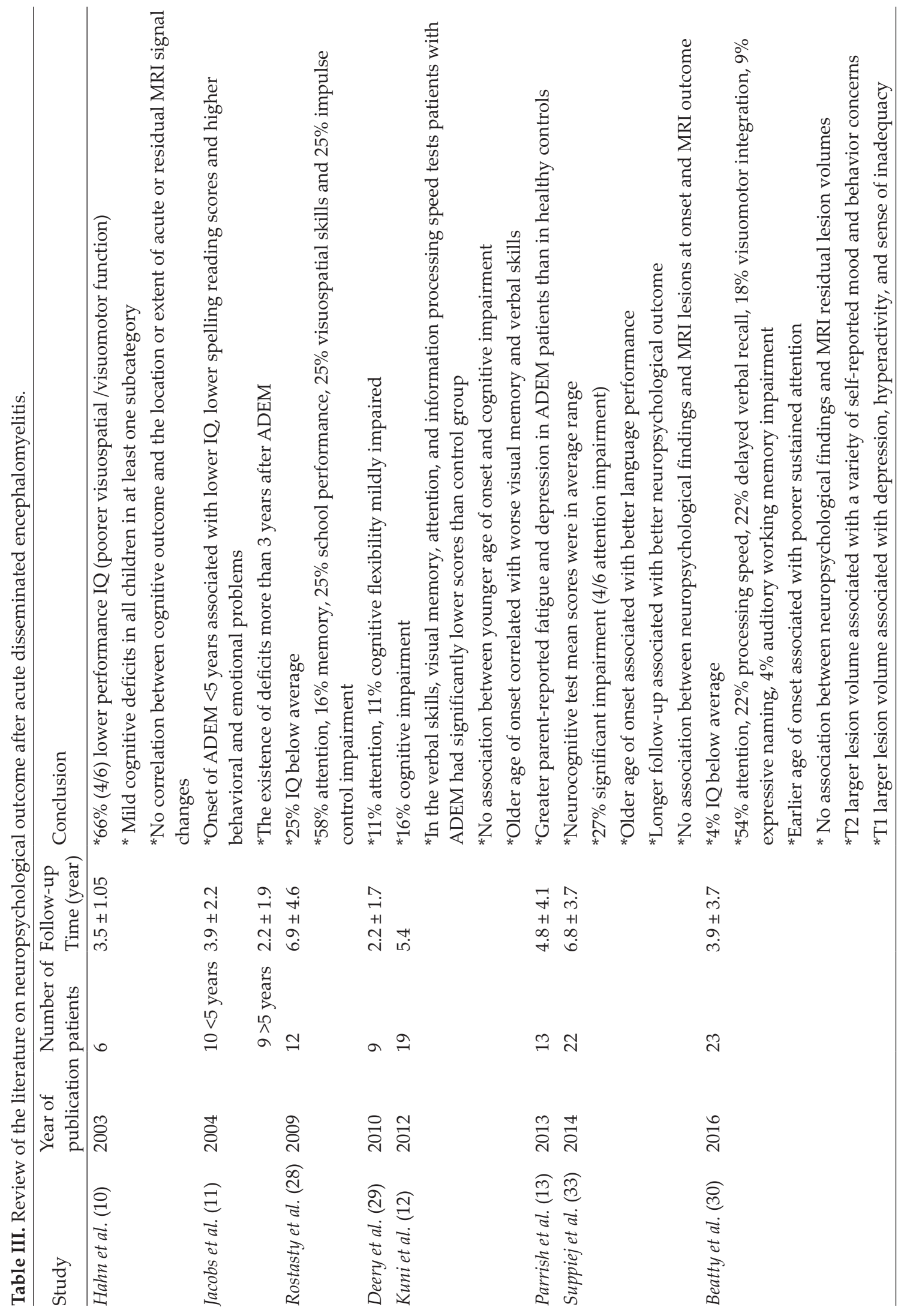




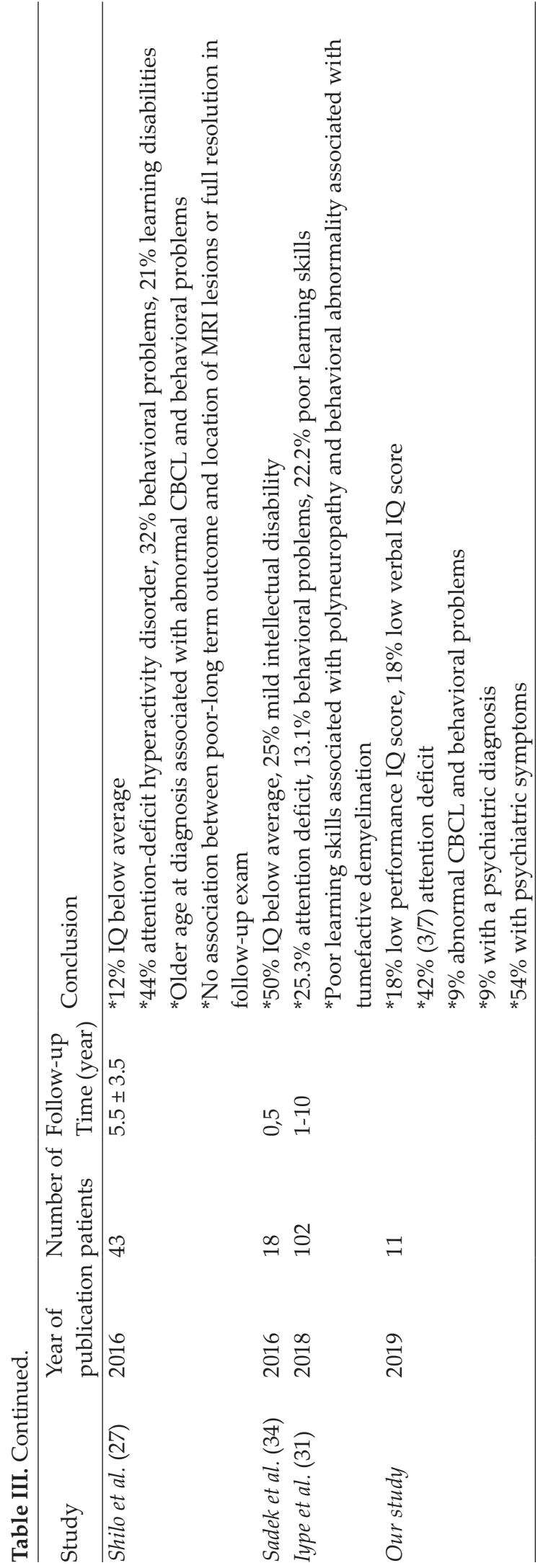

visuospatial/visuomotor skills relative to verbally mediated skills.

Jacobs et al. ${ }^{11}$ emphasized that the children who were diagnosed with ADEM before 5 years of age had more severe behavioral and emotional problems. Beatty et al. ${ }^{30}$, reported a psychiatric abnormality in $20-40 \%$ of 23 cases and pointed out that having the disease at a younger age is a risk factor for intellectual disability and behavioral disorders. White matter, which is the most commonly affected area in ADEM, shows the fastest development in early childhood, and axonal myelinization and synaptic density are closely correlated with functional development. ${ }^{29}$ In particular, development of axonal myelinization and synaptic density were prominent between 3-7 years. ${ }^{34}$ For this reason, the relationship between the age at the diagnosis of ADEM and the neurocognitive status is significant, and there is another study suggesting that cases diagnosed at a younger age have poorer outcomes in terms of speech function. ${ }^{32}$ In our study, their first diagnostic ages of cases with neuropsychiatric symptoms ranged between 4-14 years, one of them was younger than 5 years.

In our group, we found that basal ganglia involvement at the beginning of the disease might be associated with attention problems at follow-up. In previous studies, any relationship between late neurocognitive dysfunction and lesion localizations have not been reported. ${ }^{10,27,32}$ In our study, attention deficit or cognitive symptoms were most frequent in cases with basal ganglia involvement. There are many studies pointing out abnormalities of basal ganglia in children with ADHD. ${ }^{35-37}$ Similarly, low verbal IQ scores and language problems have been reported in children with basal ganglia lesions. ${ }^{36-39}$ In accordance with the literature, we couldn't find any relationship between neuropsychiatric symptoms and resolution of lesions on follow-up MRI. ${ }^{27,30,32}$

The limitations of this study are: (1) inclusion of a small sample size, (2) lack of a control group, (3) lack of application of CPT to all 
cases. Despite the limited number of cases in our study, comprehensive cognitive and psychiatric evaluations were performed. In conclusion, psychosocial and cognitive problems were detected in $54.5 \%$ of cases with ADEM. In particular, cases with basal ganglia involvement should be followed carefully in terms of attention and cognitive problems.

\section{REFERENCES}

1. Jayakrishnan MP, Krishnakumar P. Clinical profile of acute disseminated encephalomyelitis in children. J Pediatr Neurosci 2010; 5: 111-114.

2. Menge T, Hemmer B, Nessler S, et al. Acute disseminated encephalomyelitis: an update. Arch Neurol 2005; 62: 1673-1680.

3. Pohl D, Alper G, Van Haren K, et al. Acute disseminated encephalomyelitis: updates on an inflammatory CNS syndrome. Neurology 2016; 87(9 Suppl 2): S38-S45.

4. Krupp LB, Banwell B, Tenembaum S; International Pediatric MS Study Group. Consensus definitions proposed for pediatric multiple sclerosis and related disorders. Neurology 2007; 68(16 Suppl 2): S7-S12.

5. Khong PL, Ho HK, Cheng PW, Wong VCN, Goh W, Chan FL. Childhood acute disseminated encephalomyelitis: the role of brain and spinal cord MRI. Pediatr Radiol 2002; 32: 59-66.

6. Tenembaum S, Chamoles N, Fejerman N. Acute disseminated encephalomyelitis: a long-term followup study of 84 pediatric patients. Neurology 2002; 59: 1224-1231.

7. Visudtibhan A, Tuntiyathorn L, Vaewpanich J, et al. Acute disseminated encephalomyelitis: a 10-year cohort study in Thai children. Eur J Paediatr Neurol 2010; 14: 513-518.

8. Anlar B, Basaran C, Kose G, et al. Acute disseminated encephalomyelitis in children: outcome and prognosis. Neuropediatrics 2003; 34: 194-199.

9. Tenembaum S, Chitnis T, Ness J, Hahn JS; International Pediatric MS Study Group. Acute disseminated encephalomyelitis. Neurology 2007; 68(16 Suppl 2): S23-S36.

10. Hahn CD, Miles BS, MacGregor DL, Blaser SI, Banwell BL, Hetherington CR. Neurocognitive outcome after acute disseminated encephalomyelitis. Pediatr Neurol 2003; 29: 117-123.
11. Jacobs RK, Anderson VA, Neale JL, Shield LK, Kornberg AJ. Neuropsychological outcome after acute disseminated encephalomyelitis: impact of age at illness onset. Pediatr Neurol 2004; 3: 191-197.

12. Kuni BJ, Banwell BL, Till C. Cognitive and behavioral outcomes in individuals with a history of acute disseminated encephalomyelitis (ADEM). Dev Neuropsychol 2012; 37: 682-696.

13. Parrish JB, Weinstock-Guttman B, Smerbeck A, Benedict RHB, Yeh EA. Fatigue and depression in children with demyelinating disorders. J Child Neurol 2013; 28: 713-718.

14. Tan A, Hague C, Greenberg BM, Harder L. Neuropsychological outcomes of pediatric demyelinating diseases: a review. Child Neuropsychol 2018; 24: 575-597.

15. Burton KLO, Williams TA, Catchpoole SE, Brunsdon RK. Long-term neuropsychological outcomes of childhood onset acute disseminated encephalomyelitis (ADEM): a meta-analysis. Neuropsychol Rev 2017; 27: 124-133.

16. Hynson JL, Kornberg AJ, Coleman LT, Shield L, Harvey AS, Kean MJ. Clinical and neuroradiologic features of acute disseminated encephalomyelitis in children. Neurology 2001; 56: 1308-1312.

17. Kaufman J, Birmaher B, Brent D, et al. Schedule for affective disorders and schizophrenia for school-age children-present and lifetime version (K-SADS-PL): initial reliability and validity data. J Am Acad Child Adolesc Psychiatry 1997; 36: 980-988.

18. Gökler B, Ünal F, Pehlivantürk B, Kültür E, Akdemir D, Taner Y. Reliability and validity of schedule for affective disorders and schizophrenia for school age children-present and lifetime version-Turkish version (K-SADS-PL-T). Turk J Child Adolesc Ment Health 2004; 11: 109-116.

19. Savaşır I, Şahin N. Wechsler Çocuklar İçin Zeka Ölçeği Uygulama Kitapçı̆̆ı. Ankara: Türk Psikologlar Derneği, 1995.

20. Lapierre D, Braun CMJ, Le Pailleur L. A 54-point verbal-performance IQ discrepancy on the WISe-R: cognitive functioning of a child from an alternative school. Behav Neurol 1992; 5: 97-106.

21. Öy B. Çocuklar için depresyon ölçeği: geçerlik ve güvenirlik çalışması. Türk Psikiyatri Dergisi 1991; 2: 132-136.

22. Achenbach TM, Edelbrock C. Manual for the Child Behavior Checklist and Revised Child Behavior Profile. Burlington: Queen City Printers, 1983. 
23. Varni JW, Seid M, Kurtin PS. PedsQL 4.0: reliability and validity of the Pediatric Quality of Life Inventory version 4.0 generic core scales in healthy and patient populations. Med Care 2001; 39: 800-812.

24. Çakın Memik N, Ağaoğlu B, Coskun A, Üneri ÖS, Karakaya I. The validity and reliability of the Turkish Pediatric Quality of Life Inventory for children 13-18 years old. Turk J Psychiatry 2007; 18: 353-363.

25. Uneri OS, Agaoglu B, Coskun A, Memik NC. Validity and reliability of Pediatric Quality of Life Inventory for 2- to 4-year-old and 5- to 7-year-old Turkish children. Qual Life Res 2008; 17: 307-315.

26. Conners CK, Sitarenios G, Ayearst L. Conners' Continuous Performance Test Third Edition. In: Kreutzer J, DeLuca J, Caplan B. (eds) Encyclopedia of Clinical Neuropsychology. Cham, Switzerland: Springer, 2018: 1-4.

27. Shilo S, Michaeli O, Shahar E, Ravid S. Long-term motor, cognitive and behavioral outcome of acute disseminated encephalomyelitis. Eur J Paediatr Neurol 2016; 20: 361-367.

28. Rostásy K, Nagl A, Lütjen S, et al. Clinical outcome of children presenting with a severe manifestation of acute disseminated encephalomyelitis. Neuropediatrics 2009; 40: 211-217.

29. Deery B, Anderson V, Jacobs R, Neale J, Kornberg A. Childhood MS and ADEM: investigation and comparison of neurocognitive features in children. Dev Neuropsychol 2010; 35: 506-521.

30. Beatty C, Bowler RA, Farooq O, et al. Long-term neurocognitive, psychosocial, and magnetic resonance imaging outcomes in pediatric-onset acute disseminated encephalomyelitis. Pediatr Neurol 2016; 57: 64-73.

31. Iype M, Ts A, Kunju PM, Saradakutty G, Sreedharan $\mathrm{M}$, Ahamed SM. Factors related to long term motor, behavioral, and scholastic outcome in children with acute disseminated encephalomyelitis. Pediatr Neurol 2018; 89: 49-57.
32. Suppiej A, Cainelli E, Casara G, Cappellari A Nosadini M, Sartori S. Long-term neurocognitive outcome and quality of life in pediatric acute disseminated encephalomyelitis. Pediatr Neurol 2014; 50: 363-367.

33. Sadek AA, Mohamed MA, Abou-Taleb A, Mohammed MI. Pattern and Outcome of Acute Disseminated Encephalomyelitis (ADEM) in children: experience in a tertiary center, Upper Egypt. Electron Physician 2016; 8: 2679-2685.

34. Tideman E, Gustafsson JE. Age-related differentiation of cognitive abilities in ages 3-7. Pers Individ Dif 2004; 36: 1965-1974.

35. Nakao T, Radua J, Rubia K, Mataix-Cols D. Gray matter volume abnormalities in ADHD: voxelbased meta-analysis exploring the effects of age and stimulant medication. Am J Psychiatry 2011; 168 : 1154-1163.

36. Verma S, Kushwaha S. Intelligence and attention deficit hyperactivity disorder. J Psychol Res 2016; 11: 417-425.

37. RivaD, Taddei M, BulgheroniS. Theneuropsychology of basal ganglia. Eur J Paediatr Neurol 2018; 22: 321 326.

38. Lewis JD, Fonov VS, Collins DL, Evans AC, Tohka $\mathrm{J}$; for the Brain Development Cooperative Group and the Pediatric Imaging, Neurocognition, and Genetics Study. Cortical and subcortical T1 white/ gray contrast, chronological age, and cognitive performance. Neuroimage 2019; 196: 276-288.

39. Peterson RK, McDonald KP, Vincent M, Williams TS, Dlamini N, Westmacott R. Characterizing language outcomes following childhood basal ganglia stroke. Appl Neuropsychol Child 2019: 1-12. 